\title{
Procedure Route of Administration
}

National Cancer Institute

\section{Source}

National Cancer Institute. Procedure Route of Administration. NCI Thesaurus. Code C117524.

The means of entry through which the procedure is taken into the body. 